\title{
Post Launch Calibration and Testing of the Advanced Baseline Imager on the GOES-R Satellite
}

\author{
William Lebair ${ }^{\mathrm{a}}$, C. Rollins ${ }^{\mathrm{b}}$, John Kline ${ }^{\mathrm{b}}$, M. Todirita ${ }^{\mathrm{c}}$, J. Kronenwetter ${ }^{\mathrm{d}}$ \\ aNASA Goddard Space Flight Center, Code 910, Greenbelt, MD 20771

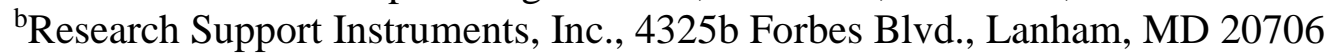 \\ ${ }^{\mathrm{c}}$ NOAA/NESDIS GOES-R Flight Project, Greenbelt, MD 20771 \\ ${ }^{\mathrm{d} C h e s a p e a k e ~ A e r o s p a c e, ~ P . O . ~ B o x ~ 567, ~ G r a s o n v i l l e, ~ M D ~} 21638$
}

\begin{abstract}
The Geostationary Operational Environmental Satellite R (GOES-R) series is the planned next generation of operational weather satellites for the United State's National Oceanic and Atmospheric Administration. The first launch of the GOES-R series is planned for October 2016. The GOES-R series satellites and instruments are being developed by the National Aeronautics and Space Administration (NASA). One of the key instruments on the GOES-R series is the Advance Baseline Imager (ABI). The ABI is a multi-channel, visible through infrared, passive imaging radiometer. The ABI will provide moderate spatial and spectral resolution at high temporal and radiometric resolution to accurately monitor rapidly changing weather.

Initial on-orbit calibration and performance characterization is crucial to establishing baseline used to maintain performance throughout mission life. A series of tests has been planned to establish the post launch performance and establish the parameters needed to process the data in the Ground Processing Algorithm. The large number of detectors for each channel required to provide the needed temporal coverage presents unique challenges for accurately calibrating $\mathrm{ABI}$ and minimizing striping.
\end{abstract}

This paper discusses the planned tests to be performed on ABI over the six-month Post Launch Test period and the expected performance as it relates to ground tests.

\section{OVERVIEW}

The next generation of United States' Geostationary weather satellites will begin with the launch of the GOES-R satellite in October 2016. The acronym GOES refers to Geostationary Operational Environmental Satellite, and the R-series comprises GOES-R, -S, -T, and -U, all currently in varying stages of manufacture and assembly. The current paper discusses planned post-launch, on-orbit testing for one of the primary instruments, the Advance Baseline Imager (ABI).

The ABI is a scanning 16-channel multispectral imager spanning the visible, near infrared, short wave infrared, midwave infrared and long wave infrared regions of the spectrum. The detector arrays span approximately one degree in the North-South direction at varying resolution ranging from $1 / 2$ kilometer for the 0.64 -micron visible channel to 2 kilometers for the longer wavelength infrared channels. In normal operation, scanning is performed in a series of slightly overlapping west-to-east scans, each successive scan being more southerly than the preceding scan. The number of such scans depends on the synoptic area being collected and ranges from two for storm tracking to several for continentalsized regions to more than twenty for full Earth disk coverage.

In addition to the large number of detectors in the north-south direction, each array also has several columns of detectors in the east-west direction, providing several-fold redundancy. Detector selection can be implemented column-by-column (designated "column mode') or in a true, random access, detector-by-detector best-detector-selection process (designated "best detector select" or BDS mode). For diagnostic purposes, it is also possible to collect from all detectors (designated "all column mode"). In addition to the complexity arising from the number of detectors, each array's performance can also be optimized through adjustment of various clocks and biases.

Management of this complex instrument involves characterization, optimization, and calibration through a series of procedures and tests. While many of these activities can be and have been conducted on the ground during instrument integration and test, the ultimate performance of the instrument must be established on station in the orbital environment. 
In addition, aging effects over time may require subsequent on-orbit adjustments. The subsequent pages of this paper discuss the planned post-launch testing that will be carried out once the $\mathrm{ABI}$ instrument arrives on orbit.

\section{POST-LAUNCH TESTS}

The initial phase of post-launch testing is geared to demonstrating the health and safety of the instrument. This phase begins with demonstration of the solar avoidance algorithm, and then performs an initial detector performance screening, using on-board reference sources, to support selection of the best detectors. This phase also sees the initialization of scanner and scan encoder performance tracking. The process then continues with radiometric testing including basic radiometric calibration, demonstration of calibration stability, noise performance, dynamic range, and repeatability, as well as the execution of initial observations with desert scenes for characterization of the VNIR channels. These initial measurements also provide the starting point for radiometric performance trending over the life of the instrument. Subsequent tests verify image navigation and registration (INR), and image quality. The full complement of post launch tests is described below, in the order in which they first appear in the post-launch test cycle.

\subsection{Solar Avoidance}

The purpose of this test is to confirm that the $\mathrm{ABI}$ on-board solar avoidance algorithms function properly prior to opening the Optical Port Cover and risking a solar exposure event. Prior to opening the Optical Port Cover (OPC), the $\mathrm{ABI}$ is powered and set to a diagnostic mode.

The ABI autonomously adjusts the scan pattern to avoid scanning within a ground definable angle from the center of the Sun. To do so, flight software computes an exclusion zone. The exclusion zone is a region defined around the sun that is designed to be sufficiently large so that swath truncation results in more than 0.5 degrees margin for the edge of the FOV reaching the edge of the sun. That is to say that the swath truncation results in the edge of the FOV getting no closer than 0.5 degrees from the edge of the sun when bright object avoidance is applied. This exclusion zone is a circular area centered on the sun at any given point in time (see Figure 1). However, since the sun moves with respect to the ABI during the swath duration, the actual exclusion zone for a single swath is an oval-shaped area. If it is determined that any part of the swath will intersect this exclusion zone, the swath is truncated to protect $\mathrm{ABI}$ from viewing the sun.

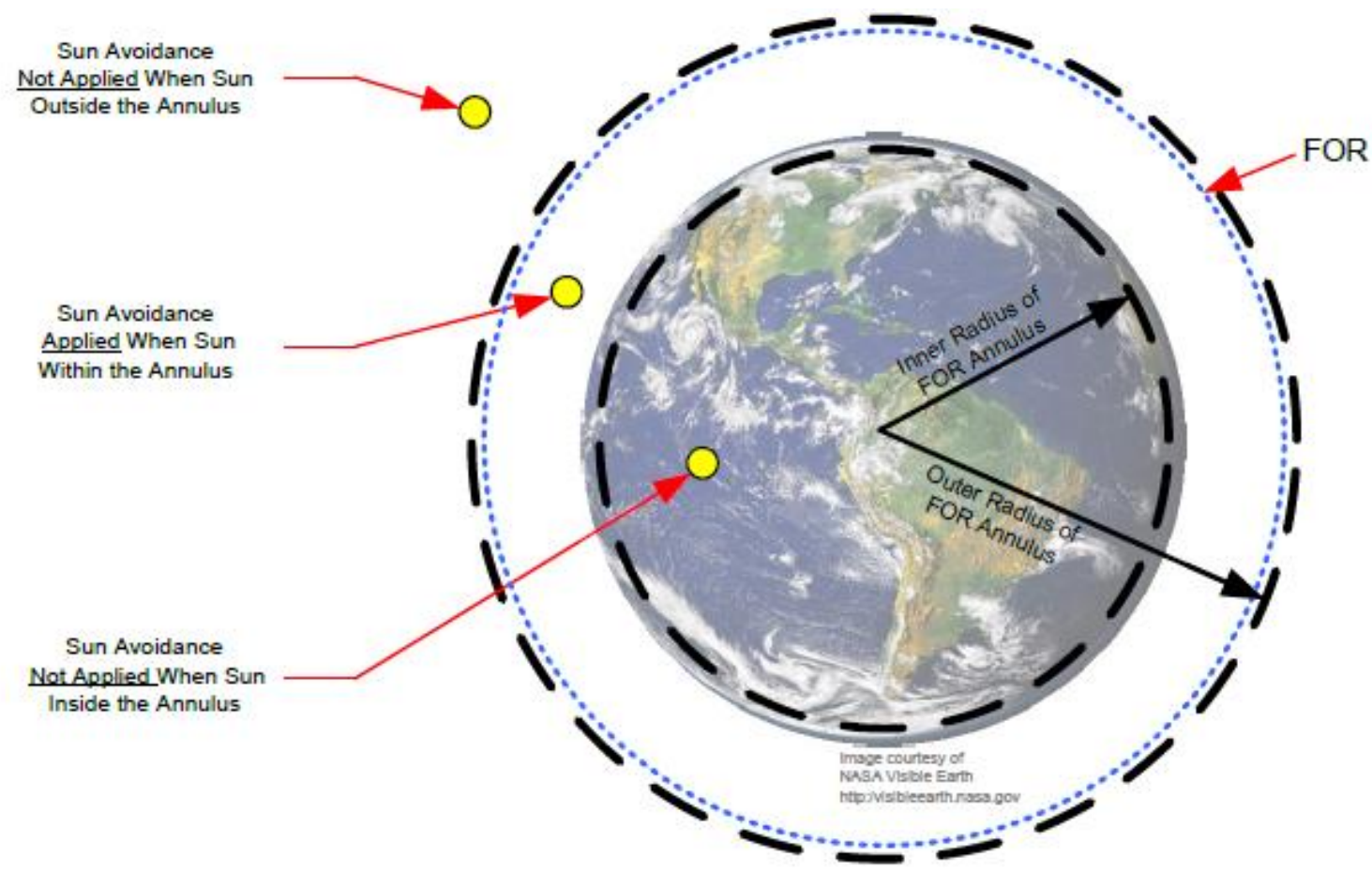

Figure 1: Sun Exclusion Zone for Sun Avoidance Algorithm

When the sun is within the FOR of the ABI, a series of scans is performed with the Sun Avoidance Autonomous Function enabled. Because the Sun is in the field of regard, it is expected that the avoidance algorithm will truncate those scans that approach too close to the Sun. If the Sun's closest approach doesn't result in a truncated scan, then the 
algorithm's Sun Avoidance parameter is adjusted to ensure a swath will be truncated. This data collection is repeated for at least 2 separate sun locations (e.g. as the sun has moved from the east side to the west side of the ABI FOR).

To complete the test, the scan patterns are examined to confirm that the correct swaths were skipped or shortened to avoid the sun. Once proven, ordinary scan operations can be started following the OPC deployment.

\subsection{Detector Screening and Best Detector Selection}

Each color channel of the ABI is logically a line array that is swept across the scene to build up a two dimensional image. In fact, however, each channel is made up of several columns of detectors to provide redundancy. For each row of the array, a detector can be selected from one of the columns to populate the logical line array. This allows selection of the best performing detector per row, to optimize performance or to avoid a detector that exhibits any undesirable properties.

During ground testing, an optimized set of detectors will have been identified, but it is expected that individual-detector properties may change behavior with age and with each cool-down cycle. The purpose of the detector screening test is to determine if any individual detectors have changed performance after the on-orbit cool down and to identify detectors from the row of redundant detectors to use in their place.

The screening procedure uses a series of well-defined image collections to build a performance data set for each detector. Different criteria are of importance for the VNIR reflective channels (those intended to measure spectral albedo) and for the infrared thermal channels (those intended to measure temperature). Calibration of the reflective channels is performed using observations of an on-board solar diffuser and observations of dark space. Calibration of the thermal channels is performed using observations of an on-board thermal reference and observations of cold space. Stability of the thermal channels is determined using long-duration images of the on-board thermal target while stability of the reflective channels is determined using long-duration views of stable ground scenes. The screening test is designed to collect all of these in a sequence.

First, VNIR images are collected while staring alternately at the Solar Calibration Target (SCT) and at space. Second, thermal images are collected from the Internal Calibration Target (ICT) and from space scenes while adjusting the focal plane arrays applied bias voltages. Third, a collection executes a long duration ( 30s) "stare" at the ICT and space. Finally, VNIR data are collected while staring alternately at an Earth target and at space. A full Earth disk image is collected and analyzed to look for a large area on the Earth (goal of greater than 1.0 degree NS by 2.0 degrees EW) providing large, time-stable radiance (goal of greater than 50\% albedo for all 6 VNIR channels). NOTE: The area does not have to have uniform radiance over the $1.0 \mathrm{deg}$ x $2.0 \mathrm{deg}$ area, but should be time-stable over a period on the order of 5 minutes. Once the area is identified, the instrument stares at the Earth target and space for 2.4 seconds each (swath time in instrument-level calibration) with nominal biases and integration times.

An automated ground-station processing algorithm is used to evaluate performance parameters including gain and gain stability, offset and offset stability, and noise performance. The output of this algorithm is a recommended best-detectorselect (BDS) map that can be uploaded to the instrument at the discretion of the operators.

\subsection{Scan Encoder Fine Track Error Trending}

The purpose of this test is to track changes in commanded versus executed scan mirror positioning. The fine-scale relationship between commanded scan mirror position and actual position depends on encoder calibration coefficients and on environmental and/or aging factors. The purpose of this first scanner encoder test is to perform initial calibration of the fine track error (FTE) of the ABI scan system and trend over on-orbit conditions. To track this performance, a small number of pre-defined pointing scenarios have been constructed. Returned telemetry provides information on the execution of these commands. Results are trended over time so that new coefficients can be loaded when necessary.

\subsection{IR Radiometric Calibration Stability}

The ABI has a requirement on each thermal channel (those with a wavelength greater than 3 microns) that while viewing a stable $300 \mathrm{~K}$, the calibration-to-calibration repeatability be less than 0.2 Kelvin. To demonstrate this, the ABI executes 15-minute "Mode-3" timelines consecutively over a 24 hour period. Among other things, this timeline provides a 3 CONUS images and a blackbody reference look every 15 minutes. CONUS images taken immediately before and after a reference look are spatially averaged and differenced in radiance space, then converted to an effective delta-temperature. The successive differences are then tracked over time using the RSS of consecutive pairs. Analysis is conducted to determine the drift in calibration coefficients necessary for a $0.2 \mathrm{~K}$ brightness temperature shift for each channel. 


\subsection{Alignment Assessment}

This post-launch test determines and corrects for post-launch misalignment offsets between ABI and the spacecraft attitude determination system (see also "Image navigation and registration performance assessment tool set for the GOES-R Advanced Baseline Imager and Geostationary Lightning Mapper" in these proceedings). Specifically, the test performs assessments of Landsat ground control points in Level 1B imagery to determine 3-axis navigation offsets. Star positions in the ABI imagery are not used for navigation updates; this approach effectively ties the navigated line of sight) LOS to the $\mathrm{s} / \mathrm{c}$ attitude determination frame. The Landsat ground control point residuals represent the difference between the LOS and the s/c attitude determination frame. The residuals are used to construct diurnal profiles, and lineof-site motion compensation (LMC) coefficients are determined from the bias component of the profiles. Navigation analysis is repeated with the landmark-analysis-based delta LMC coefficients to confirm the diurnal profile has been compensated to within the uncertainty bounds of the Landsat ground control point matching analysis.

Navigation analysis is repeated with the landmark analysis based delta LMC coefficients to confirm the diurnal profile has been compensated to within the uncertainty bounds of the Landsat ground control point matching analysis.

A separate post launch test will further refine the LMC coefficients using ABI stars, after the initial misalignment is corrected by this PLT to facilitate autonomous ABI star acquisition.

\subsection{IR NEdT and Dynamic Range Characterization}

This test sequence establishes the beginning of life noise-equivalent delta temperature (NEdT), dynamic range, and Quantization Step Size (QSS) in the thermal channels of the ABI.

Noise:

Long duration measurements ( 2 seconds) of space and of the ABI internal calibration target (ICT) are collected. Noise terms are calculated in units of temperature.

\section{Dynamic Range:}

Dynamic range is determined by measuring the $\mathrm{ABI}$ response in digital counts as a function of integration time while staring at the ICT. The integration time is proportional to the instrument response, making the radiance at the top of the dynamic range easy to calculate. There is an underlying assumption of the equivalence in accumulated signal between increased integration time and increased radiance. This equivalence has been separately demonstrated to high accuracy in ground testing.

QSS:

QSS is calculated using the dynamic range data and the downlink bit data either as the ratio of the largest radiance to the largest digital count value or as the slope of the radiance-versus-counts curve. The latter is valid owing to the high degree of linearity of the ABI detectors.

\subsection{IR Radiometric Repeatability Characterization}

The ABI has repeatability requirements with respect to adjacent pixels, between swaths, between channels, and between successive images. The specifications for these are stated relative to the SNR (reflective channels) or NEDT (thermal channels). For each of these, there is an associated noise equivalent digital number (NEDN) that states the noise level as measured in digital counts. For thermal channels, the pixel-to-pixel and swath-to-swath repeatability requirements, performance level must be less than the NEDN. Between thermal channels and between images, the repeatability must be less than $0.2 \mathrm{~K}$.

The measurement consists of collecting imagery of the internal calibration target in a manner that mimics typical operational use. This typical use consists of capturing a CONUS image every five minutes, interleaved with collecting an Earth full disk every 15 minutes.

For pixel-to-pixel repeatability, the local RMS row deviation relative to the sub-image mean of every 3-row-by-10column sub-image is calculated. This RMS deviation is averaged over a sufficient number of images to establish the performance requirement at the $90 \%$ confidence level. By doing this for every 3 -by 10 sub-image, adherence to the requirement is verified everywhere over the full disk.

For swath-to-swath repeatability, boundaries are identified in CONUS-sized images where adjacent rows come from a north swath and a south swath respectively. The row averages are calculated for the more northern and more southern 
rows respectively. The north-south difference between the means is then RMS averaged over a sufficiently large number of boundaries to establish performance at the $90 \%$ confidence level.

For channel-to-channel repeatability, average temperature is calculated over successive CONUS-sized images in each of two channels. The result is RMS averaged over a sufficiently large number of images to assure performance at the $90 \%$ confidence level. This process is repeated between all possible thermal channel pairs.

For image-to-image repeatability, the average temperature is calculated over successive CONUS-sized images. Mean temperature differences are then calculated over successive image pairs. These differences are the RMS averaged over a sufficiently large number of images to assure performance at the $90 \%$ confidence level. This process is repeated for each of the thermal channels.

\subsection{VNIR Radiometric Calibration Trending}

Solar calibrations are conducted every two days, for twenty days, then with prescribed decreasing frequency for the balance of the test phase. The goal is to provide baseline trending measurements for solar calibration performance and characterize calibration stability.

Solar calibrations consist of six prolonged stares (one for each VNIR channel) at the on-board solar-diffuser calibration target, sandwiched with six prolonged stares at cold space. The resulting data set provides two-point calibration with good statistics for selected detector in every row of the detector array for each VNIR channel. The initial results are compared with ground calibration data to verify in-family performance. Successive results provide longer term trending and provide motivation for the planned decrease in calibration frequency.

\subsection{Desert VNIR Monitoring}

Three "vicarious calibration" desert targets have been identified to provide ground-truth validation of the on-board calibration process, provide trending of detector performance for all operational VNIR detectors, and to provide an alternate calibration methodology in the event of the failure of the on-board solar calibration target. The three identified targets are: 1) Sonoran Desert; 2) White Sands Missile Range; 3) Uyuni Salt Flats, Bolivia (see Figure 2). For each of these targets, a series of six north-south scans is conducted so as to sequentially place each of the six VNIR channels over the desired ground track. The ground track for each of the three identified sites is approximately $200 \mathrm{~km}$ in length. The observations will be carried out within \pm 7.5 minutes of local solar noon at each target site.

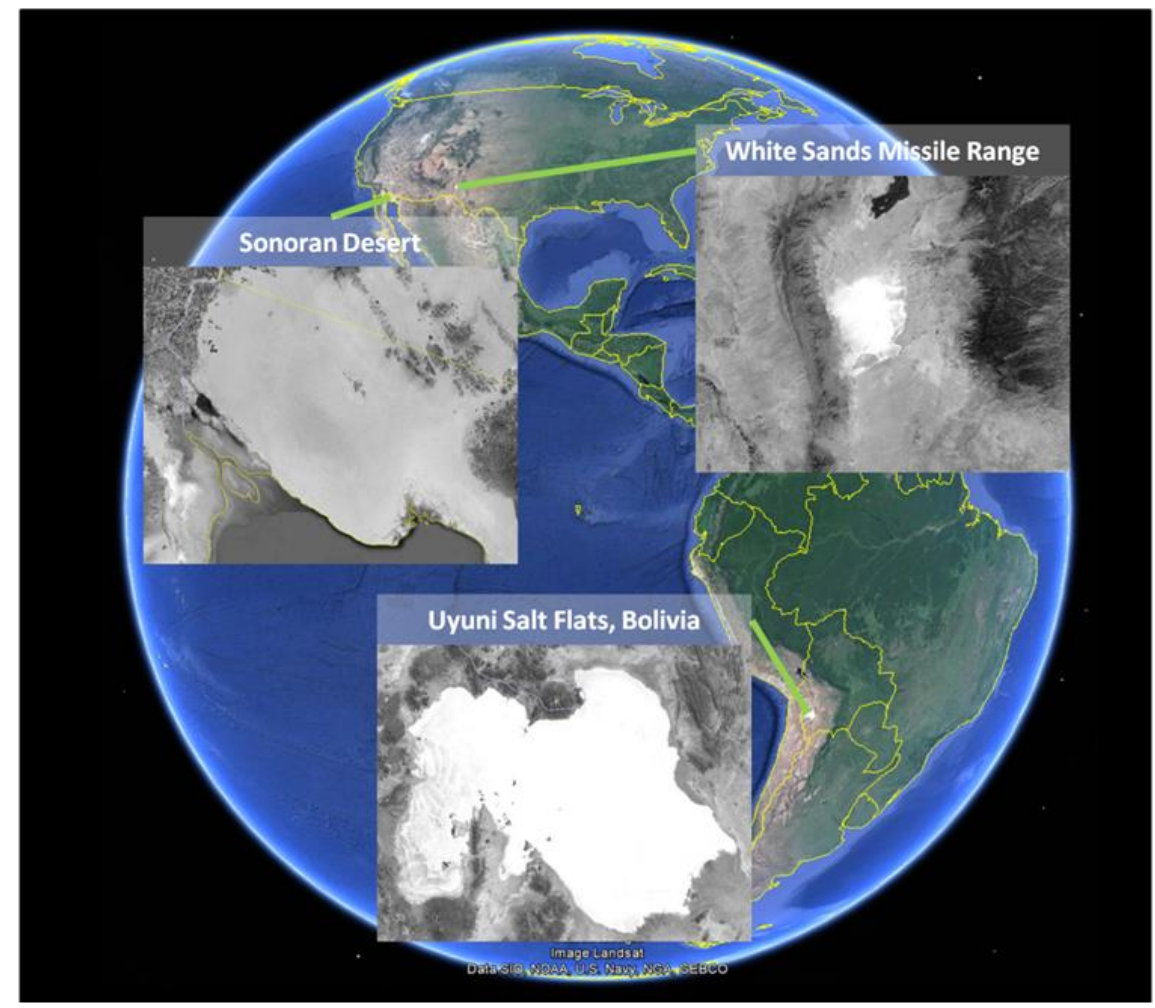

Figure 2: Desert validation targets for routine PLT/PLPT ABI NSS collections. 


\subsection{System Linearity}

This test steps the integration time in constant increments, covering just over the entire operational dynamic range for each channel. While stepping the VNIR channels, the instrument stares at the solar calibration target. While stepping the thermal channels, the instrument stares at the thermal internal calibration target. Because the 3.9 micron channel is set to have significantly higher sensitivity, a third step size is used uniquely for that channel. A linear fit is conducted for the collected data, to demonstrate less than $1 \%$ nonlinearity.

\subsection{Coherent Noise}

Synthetic full disk images are collected while staring at the internal blackbody target interleaved with space observations using a timeline that mimics actual full disk collections. 2-D Fourier transforms are used to identify any spatial frequencies that contribute at a level exceeding $25 \%$ of the NEDN. Results are compared to ground measurements to ensure in-family performance

\subsection{Spatial Uniformity Characterization over Field of Regard}

The intent of this test is to measure the beginning of life spatial variation in channel response over the field of regard of the instrument and thereby confirm that the scan mirror emissivity/reflectivity coefficients are valid. The process begins with a blackbody calibration and is then followed by eight looks to space at locations equally spaced around the Earth. Each space look lasts 25 seconds to provide high quality statistics. The process is repeated once every four hours for 24 hours. The data are evaluated by comparing calibrated detector samples across array and at each space location. Should the uniformity exceed the required $0.3 \%$, then a spatial uniformity re-calibration is conducted.

\subsection{Line of Sight Estimation Filter Performance Characterization and Tuning}

This two-part test is designed first to characterize the performance of the (ground based) line of sight estimation filter and second to characterize the line-of-site diurnal profile for use in computing tuning parameters for the filter. Star measurements are conducted for a predetermined set of stars. The stars are navigated to a fixed grid using current navigation parameters. Star position navigation errors as well as measurement noise parameters are extracted for use in the LOS estimation filter.

\subsection{LMC Coefficient Generation}

Line of Sight Motion Compensation (LMC) parameters are used on the instrument to adjust line of sight pointing for diurnal offsets of the instrument mounting interface to the spacecraft. The parameters are also used to characterize the instrument mounting alignment offsets resulting from launch loads. This test uses extracted Line of Sight diurnal profile data based on LOS estimation filter states to compute the average line of sight motion compensation parameters. An average set of LMC parameters is computed over daily and seasonal diurnal profile variations. If the average value of the LMC parameters is sufficient to allow ABI to meet pointing error allocations, then the average static values can be used on a permanent basis. If the seasonal variation in diurnal profile is such that the ABI will not meet pointing error allocations, then additional sets of LMC parameters will be necessary to meet performance over the seasonal variation.

\subsection{Co-registration Characterization Analysis (MWIR/LWIR offset)}

Channel-to-channel registration error, or co-registration, is the difference in navigation between spectral channels for any given pixel in the same frame. The co-registration error between the MWIR and the LWIR channels is required to not exceed 11.2 microradians.

The test consists of collecting star scenes with both the MWIR 3.9-micron and one of the LWIR channels (optimal LWIR channel choice may depend on the selected stars). Offset in line of sight is extracted between mid-wave and longwave IR focal plane modules using star measurement residuals.

\subsection{INR EPP Deployment Assessment; EPP Stowed vs deployed}

The purpose of this test is to assess ABI Image Navigation and Registration (INR) performance before and after the Earth Pointing Platform (EPP) launch locks are released and determine if there is a performance change in INR when the EPP is released from its launch locked configuration.

24 hours of ABI imagery collected on a local noon to local noon time basis are to be assessed prior to EPP deployment and after EPP deployment. INR assessments are performed using Landsat ground control points and image to image tiepoints in Level 1B processed imagery. Navigation, within frame registration, frame-to-frame registration, swath-to-swath 
registration and channel-to-channel registration assessments will be made with Level 1B images using control points and tie-points in the images.

- Navigation compares the image with the reference control point.

- Within frame compares the distances between pairs of control points in the image with their reference control points

- Frame-to-Frame compares the same control point or tie- point in subsequence frames

- Swath-to-Swath compares a control point or tie-point in consecutive swaths

- Channel-to-Channel compares a control point or tie-point in different spectral channels (bands).

Measured ABI navigation and registration performance comparisons are made for imagery collected before and after EPP deployment.

\subsection{Spatial Resolution Characterization}

This test uses lunar imagery to measure the beginning of life modulation transfer function (MTF) performance and confirm that the measurements are within family and measurement error of the pre-launch performance baseline. Additionally, these measurements may be used to determine whether the ABI focus needs to be adjusted on-orbit. The lunar edge is used as an on-orbit MTF target because it has a "sharp" edge, there is large brightness contrast between the lunar disk and the surrounding space, and 3 a sub-pixel Edge-Spread Function (ESF) can be assembled either from a single lunar image or from a concatenation of several images. The spatial derivative of the ESF is the Line-Spread Function (LSF), and the MTF is the normalized magnitude of the Fourier Transform of the LSF. The ABI MTF will be measured in both the East-West and North-South directions using the moon.

Sequential image swaths are taken, sweeping west to east (the operational scan direction). The placement of the swaths relative to lunar latitude is adjusted so as to give multiple images each for a northerly detector region aligned to the equator, a middle region aligned to the equator, and a southerly region aligned to the equator. Scans are taken initially at the operational scan rate, then the entire sequence is repeated at $1 / 10$ of the operational rate, to separate MTF effects due to electronics from effects due to optics. Finally a set of north-to-south scans is collected at the slower rate to extract north-south optical MTF.

Through the courtesy Mr. Masaya Takahashi, Scientific Officer at the Japan Meteorological Agency (JMA), we have been provided preliminary lunar imagery from AHI-8 (which is of very similar design and construction to the ABI). Using sub-pixel alignment methods, we can overlay multiple rows across a common region of lunar terrain so as to get excellent sampling of the lunar disk edge (see Figure 3). The resultant MTF values are found, within measurement uncertainty, to meet or exceed the ABI requirements. Note that these data were derived from early imagery, prior to the AHI-8 becoming fully operational.

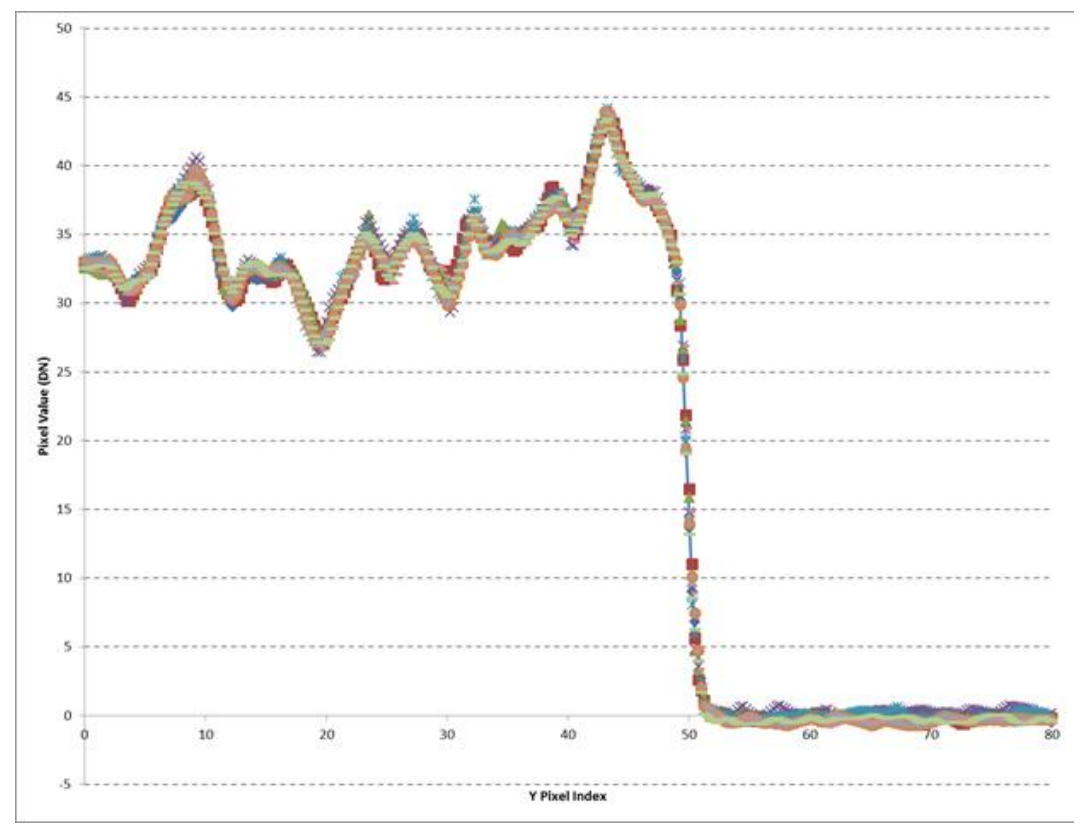

Figure 3: Edge sampling in early AHI-8 lunar images. 


\subsection{Lunar Trending for Performance}

Lunar imagery is captured when the Moon is well within the field of regard and not too close to the Earth limb. The captured imagery is used to trend VNIR calibrations over time and to evaluate relative spectral response of the scan mirrors across the field of regard. Two lunar image captures are planned per month, one for each side of the Earth. For evaluation of the scan mirror relative spectral response, at least one set of images of the moon transiting through the entire North or South end of the FOR un-occulted by the Earth will be captured.

\subsection{Stray Light Characterization in the 3.9 Micron Channel}

The principal objective of this test is to characterize and quantify stray light that may reach the ABI FPMs via a known sneak path past the field stop and scattering off the beam splitter. While a hardware approach has been developed to mitigate this stray light path, it is unable to block $100 \%$ of the stray light. It is anticipated that stray light from this path will be present for solar incidence angles between 10 and 15 degrees south of the nadir line of sight. It is expected that this effect will be present to the greatest degree in the 3.9-micron (0390) band but may be detectable throughout the VNIR. A second objective of the test is to compare the test outcome against stray light modeling predictions to verify accuracy of these modeling efforts.

Full-disk Earth images will be captured every five minutes from one hour before Spacecraft local midnight to one hour after Spacecraft local midnight every night for Beta Angles between $-14.44^{\circ}$ and $-7.5^{\circ}$. Over this time, the sun position will shift from about 19 degrees south to about 10 degrees south. Data will be navigated, radiometrically calibrated and resampled by the ground system. Images will be inspected for presence of stray light features. Image differencing will

be used between successive images to largely suppress the contribution from Earth radiance. Resulting stray light levels will be evaluated and compared against requirements.

\section{Summary}

A large and varied assortment of ABI post launch tests is planned for the pre-operational period of the GOES-R weather satellite. These tests are designed to quantify and characterize the radiometric, image quality, and navigation performance of the instrument. Results will be compared against ground measurements, where those exist, and against requirements. In some cases, these tests will be used iteratively to tune the instrument for optimal performance. In other cases, the tests will provide trending results that will confirm continued good performance or possibly indicate the need for changes in detector selection or operating parameters. The result will be a well characterized, highly optimized, state-of-the-art meteorological instrument. 